\title{
Resource review on: How to Read a Paper - the Basics of Evidence Based Medicine. Fourth edition. Oxford: Wiley-Blackwell 2010
}

\section{Terrence Shaneyfelt}

10.1136/ebm1147

Correspondence to: Terrence Shaneyfelt Birmingham Veterans Affairs Medical Center and University of Alabama School of Medicine, Birmingham, Alabama, USA; Terry.Shaneyfelt@va.gov
This book continues to impress. The first edition was excellent, the second and third even better. But the fourth edition is the best yet. Even if you own the previous editions, it is worth adding this one to your library because two new important chapters have been added on complex interventions and quality improvement (topics missing from other standard EBM textbooks).

As an EBM educator, researcher, and practitioner, I am unclear exactly for whom the book is best suited. I feel it is most suitable for those with some rudimentary understanding of EBM principles and study design but who don't want an indepth discussion of epidemiological and statistical principles. This makes it especially useful for medical students, postgraduate trainees and practicing physicians. I think it is too simplistic for researchers and seasoned EBM educators.

As Greenhalgh points out in the preface, many of the chapters are unchanged and only updated with newer references. Some chapters have been substantially revised. The chapter on searching the literature has been updated to cover many commonly used point-of-care resources and recent changes to PubMed. Unfortunately, there is neither mention of another commonly used resource (UpToDate) nor another good point-of-care resource (Essential Evidence Plus). The systematic review chapter has been updated to include the latest recommendations on reporting systematic reviews and includes recent papers on the challenges of reviewing more eclectic study types like qualitative studies and economic analyses. Finally, the chapter on implementing EBM goes beyond presenting popular methods to teach EBM and uniquely focuses on methods of changing people and organisations.

The true value of Greenhalgh's latest edition lies in its newest chapters on complex interventions and quality improvement. Studies are moving beyond single interventions (like comparing one drug to another) to include complex interventions like educational or organisational interventions. Greenhalgh describes recently developed methods to critically appraise these types of interventions. Improving the quality and safety of care often involves complex interventions, and the methods of reporting quality improvement studies are evolving. Greenhalgh nicely describes these methods in a new chapter along with the limitations of generalising the results of these types of studies.

Overall, the fourth edition maintains many of the features that have made the previous three editions popular but has additional value in two important new topics complex interventions and quality improvement studies. Greenhalgh presents a unique method of reading papers that is more fundamental than other EBM texts in that she seeks for the reader to "get their bearings" about what the paper is about prior to traditional critical appraisal. To this end, I think the book is geared more toward EBM novices than toward experts. 